\title{
Simulations of cyclic normal indentation of crystal surfaces using the bubble-raft model
}

\author{
K. J. Van Vliet and S. Suresh $\dagger$ \\ Laboratory for Experimental and Computational Micromechanics, \\ Department of Materials Science and Engineering, Massachusetts Institute of \\ Technology, Cambridge, Massachusetts 02139, USA
}

[Received 18 July 2001 and accepted in revised form 1 March 2002]

\begin{abstract}
The evolution of contact-induced deformation on the nanoscopic scale is of considerable interest in terms of both the scientific understanding of defect nucleation and the practical concern of contact damage resistance of a wide range of surfaces in engineering applications. Currently, experimental tools such as nanoindentation, atomic force microscopy, and atomic-resolution transmission electron microscopy allow quantification of nanoscale deformation and damage induced by contact at surfaces. However, none of these methods allows for in-situ visualization of atomic-level deformation during contact loading. Recently, we have employed the Bragg-Nye bubble raft to study in situ the conditions governing defect nucleation in fcc crystals subjected to nanoindentation. Although there are inherent limitations to this twodimensional model, we have found useful parallels to the mechanisms of homogeneous defect nucleation and deformation in three-dimensional fcc crystals. Such observations have the potential to guide computational models based on molecular dynamics. In this paper, we compare the characteristics of defect nucleation and slip step formation under monotonic and cyclic normal indentation using the Bragg-Nye model. We identify the atomic-level surface roughening process arising from homogeneous and heterogeneous defect nucleation and cyclic slip under repeated indentation loading. These findings provide insights into the atomic level mechanisms of cyclic slip and surface roughening during contact fatigue.
\end{abstract}

\section{$\S 1$. INTRODUCTION}

It has been postulated (for example Corcoran et al. (1997), Suresh et al. (1999) and Gouldstone et al. (2000)) that sudden displacement excursions observed during load-controlled nanoindentation of fcc crystals arise from the nucleation of dislocations beneath the nominally sharp indenter. In such experiments, a clear departure from elastic response occurs at the onset of the first displacement burst at a critical load at which the maximum shear stress beneath the indenter is of the order of the theoretical shear strength of the indented material, suggesting the possibility of homogeneous dislocation nucleation. Although transmission electron microscopy of post-indentation damage revealed the emission of dislocations from the indentation contact region (Gouldstone et al. 2000), quantification of the mechanisms

\footnotetext{
$\dagger$ Email: ssuresh@mit.edu.
} 
governing such contact-induced dislocation nucleation and kinetics in fcc crystals requires in-situ observations.

Recently, the Bragg-Nye soap bubble-raft model was used as an in-situ and quantitative experimental tool to examine the subsurface defect nucleation characteristics of fcc metals subjected to monotonic indentation (Gouldstone et al. 2001). A particularly appealing feature of the bubble-raft experimental simulation is that, despite limitations arising from its two-dimensional geometry, this model enables the creation of different atomic-level surface asperity geometries at the contact surface that cannot easily be simulated otherwise by either experiment or computational models. This bubble raft was found to be an effective system for both visualization and quantification of dislocation nucleation and motion beneath the indenter, including the evolution of surface deformation resulting from such defect nucleation.

Slip step formation at surfaces and the associated roughening of initially smooth surfaces to form intrusions and extrusions have long been known to be the primary mechanisms responsible for the nucleation of fatigue cracks in pure metal crystals (for example Suresh (1998)). Comprehensive observational evidence, obtained by recourse to detailed electron microscopy studies, is now available on how such surface roughening occurs. However, direct and in-situ studies of the evolution of damage at surfaces subjected to cyclic contact loading has thus far not been reported, particularly on the atomic level.

In this work, we employ the Bragg-Nye bubble-raft model to investigate the mechanisms of homogeneous (subsurface) and heterogeneous (surface) defect nucleation in an initially defect-free single crystal with an atomically smooth surface which is subjected to normal contact. The results obtained with this model are necessarily predicated upon an idealized case of two-dimensional geometry, and the attendant state of stress and characteristics of defect nucleation and kinetics can differ considerably from those seen in real crystals where dislocation loops rather than dipoles typically evolve during indentation. Despite this obvious limitation, the results reported here provide direct and fundamental mechanistic insights into the processes associated with contact fatigue deformation-observations which cannot be obtained through other available techniques. Indeed, it is also known from recent work (Gouldstone et al. 2001) that indentation studies using the bubble raft provide quantitative predictions of discrete plasticity events that are consistent with experimental observations of nanoindentation in fec crystals.

\section{§2. Experimental Procedures}

\subsection{Apparatus and data acquisition}

The methods of bubble-raft production used in these experiments do not deviate significantly from the original techniques; detailed descriptions of raft production have been provided elsewhere (Bragg and Nye 1947, Gouldstone et al. 2001). Essentially, uniformly sized bubbles of about $1 \mathrm{~mm}$ diameter were generated by passing pressurized air through a micropipette into a glycerine-soap solution. These bubbles naturally form a close-packed raft analogous to the $\{111\}$ plane in fcc crystals, and the in-plane orientation is determined by the boundary conditions of the raft's container. The rafts in the present work were generated to form an in-plane orientation such that the direction normal to the terminal edge (the direction of 
indentation) was $\langle 121\rangle$. The terminal edge was constructed into an 'atomically smooth surface' by bursting extraneous bubbles with a hot soldering iron.

The indenter was machined from aluminium plate of approximately $3 \mathrm{~mm}$ thickness to exhibit an included apex angle of about $139^{\circ}$ in order to approximate the geometry of a Berkovich diamond indenter in two dimensions. The indenter tip was machined to be approximately circular to idealize the blunted geometry of nominally sharp indenters used in nanoindenation experiments. Each indenter was mounted on an $x-y$ stage and partially submerged in the glycerine solution in order to avoid adhesion between the indenter and the bubbles, approximating frictionless contact. Indentation was conducted in displacement control via the motor-controlled stage, at a rate of approximately $0.3 \mathrm{~nm} \mathrm{~min}^{-1}$. Owing to the gearing of the motor, the unloading rate was slightly higher than the loading rate.

Motion of the bubbles within the raft, which contained a defect-free close-packed plane with up to 200000 atomic sites, was recorded via a high-speed high-resolution digital camera. A high rate of data acquisition $\left(10\right.$ images $\left.\mathrm{s}^{-1}\right)$ was necessary because of the high velocity of defects nucleated within the raft. Each image was recorded as a 1 Mbyte bitmap, and these images were subsequently stacked as a real-time 'movie'.

\subsection{Size scale analogy}

Bragg and Lomer (1948) extended the usefulness of this two-dimensional model by relating the attractive forces between bubbles (due to surface tension) and the repulsive forces between bubbles (due to internal pressure) to the interatomic potential of fcc crystals. They concluded that the validity of such an analogy depended on both the surface tension of the solution and the size of the bubbles. For their original solution, bubbles of approximatel y $1 \mathrm{~mm}$ diameter exhibited an experimentally measured interbubble potential that was qualitatively similar to the interatomic potential of copper. In order to exploit this connection between real fcc crystals and this twodimensional model, we have adopted the following size scale analogy: $1 \mathrm{~mm}$ in our experiments represents an atomic diameter of $0.300 \mathrm{~nm}$. According to this size scale analogy, the indenters represent contact probes with tip radii of 8,14 and $28 \mathrm{~nm}$.

\section{§. RESULTS}

The effects of the first loading cycle in a cyclic indentation experiment are illustrated in figure 1 $(a)$. Initial deformation is elastic but is followed by the 'homogeneous' nucleation of a subsurface defect along the loading axis, as predicted by the Hertzian two-dimensional continuum solution for an isotropic solid. For the range of indenter radii used in these experiments, this subsurface nucleation site is located at a depth equal to $78 \%$ of the contact half-width $a$, which is the depth corresponding to the maximum shear stress beneath a two-dimensional (cylindrical) indenter (Johnson 1985). This defect is an edge dislocation dipole. Upon nucleation, this dislocation dipole splits, with one dislocation migrating into the crystal and the other moving to the free surface. This surface deformation - one surface slip step for each dislocation dipole nucleation event - is a consistent feature of indentation in this model. If the crystal is completely unloaded after such a nucleation event, as shown in figure $1(b)$, a slip step is formed. If the crystal is not completely unloaded, the migrated defect remains as a region of localized disorder at the indenter-crystal interface.

Figure 2 shows the stages of deformation after the first complete cycle, observed during cyclic loading of the crystal with an indenter radius of $28 \mathrm{~nm}$. In this experiment, the crystal was indented until a nucleation event (including the attendant 
a

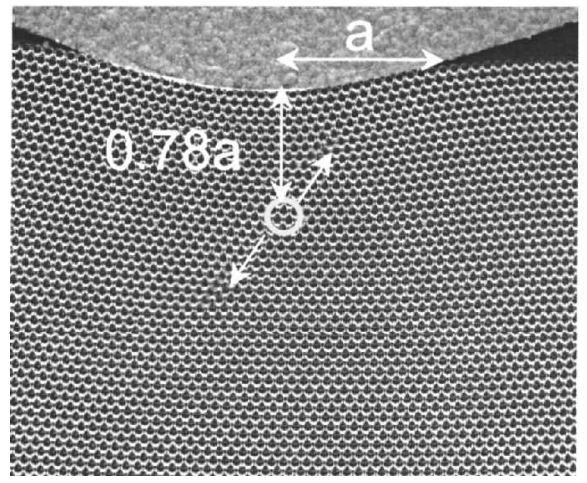

b

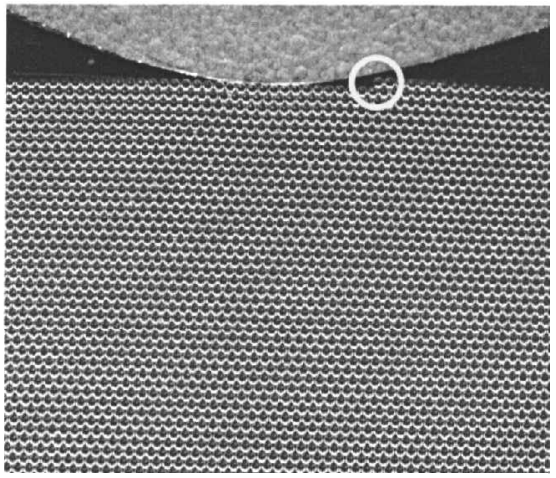

Figure 1. Evolution of dislocation activity during the first indentation loading cycle in a close-packed raft. (a) Dislocation dipole nucleation occurs at a depth $z=0.78 a$, where $a$ is the half-width of contact. This dipole splits, with one dislocation moving into the crystal and the other to the free surface. (b) Motion of the dislocation to the surface creates a slip step height of one atomic diameter. The indenter radius is $14 \mathrm{~nm}$.

$\mathrm{a}$

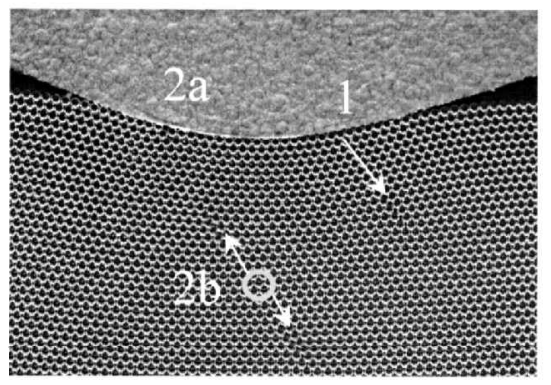

b

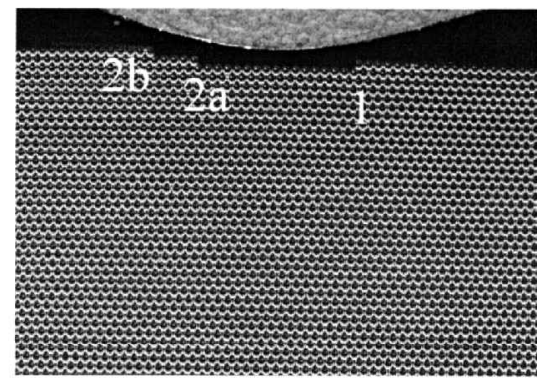

\section{c}

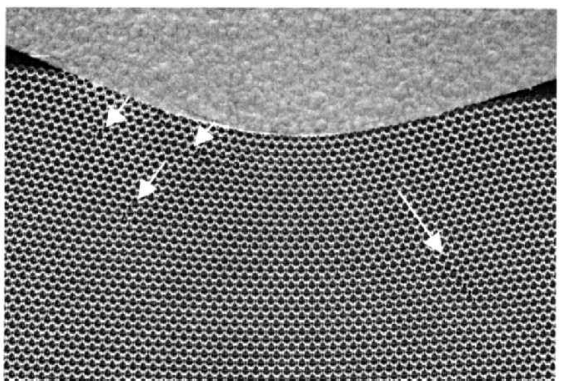

Figure 2. Surface evolution during subsequent indentation loading cycles. (a) Defects from cycles 1 and 2 are numbered. During the second loading cycle, two dislocation dipoles are nucleated internally; one dislocation from dipole 2a has migrated to the free surface. The slip step formed from cycle 1 emits defects from the surface. This activity does not suppress dislocation dipole nucleation (dipole $2 b$ ) at a depth of $0.78 a$. (b) During the fourth and subsequent loading cycles, slip steps formed during homogeneous dipole nucleation in previous cycles emit and consequently suppress further homogeneous nucleation along the loading axis. 
formation of a surface slip step) was observed to occur, immediately unloaded to complete separation between the indenter and crystal and then reloaded at precisely the same location. A maximum of six loading cycles was conducted. Upon reloading of the deformed surface shown in figure $1(b)$, the slip step then contributes its own dislocation activity, sending a dislocation directly from the surface slip step into the crystal (figure $2(a)$ ). This effect is localized, however, and does not suppress other nucleation events within the crystal. In fact, during this second loading cycle, two more homogeneous nucleation events occur along the loading axis at a depth of $0.78 a$ (only one event is shown). Figure $2(c)$ shows that, upon complete unloading, each of these nucleation events within the crystal contributes a slip step to the surface. Note that the slip step formed from nucleation during the first loading cycle reappears at the unloaded surface; although the step itself yields immediately under load, the position and the structure of the step defect are unchanged by repeated loading. The images shown in figure 2 also point to the precision and repeatability in the positioning of the indenter during the cyclic indentation experiments conducted in this work.

The above process repeats, whereby reloading of these surface slip steps initiates dislocation activity at the surface; nucleation of a new defect results in a new slip step at the surface; slip steps formed from previous nucleation events are persistent structures (figure $2(b)$ ). Note that, because the depth of nucleation increases with increasing $a$ for a fixed slip plane orientation of $30^{\circ}$ off axis, new slip steps necessarily form at increasing distances from the loading axis, and that each slip step is exactly one atomic diameter high. After several loading cycles, the dislocation activity generated from the surface slip steps dominates as the relevant nucleation mechanism, suppressing subsurface homogeneous nucleation within the crystal at the depth predicted by continuum analysis of monotonic loading (figure 2(c)). This effect appeared to be due largely to the increased interaction between the surface-generated dislocations.

Cyclic indentation experiments were conducted for several different indenter radii. As shown in figure 3 , an increase in indenter radius necessarily led to a wider spacing of surface slip steps. Otherwise, the cyclic indentation response was not a strong function of indenter radius. In fact, for all radii considered, the deformation response after three loading cycles was dominated by surface nucleation of defects from the surface slip steps. Prior to the fourth cycle, surface nucleation did not suppress subsurface dislocation nucleation.

For the same indenter radii as those examined in the above cyclic experiments, monotonic loading tests were also conducted. Here, the crystal was loaded at a constant displacement rate until several dislocation events were observed to occur. A maximum of six dislocation nucleation events was observed prior to unloading. For all indenter radii considered, the first dislocation dipole nucleation event was homogeneous and occurred at a depth of $0.78 \mathrm{a}$. However, the crystal was not unloaded and, thus, the presence of the resulting surface slip step resulted in a localized region of disorder instead of a well-defined slip step. Upon continued loading, subsequent subsurface nucleation events occurred along the loading axis, also at a depth of $0.78 a$, and the resulting surface displacements were also constrained to a localized region of disorder under continued loading. This process repeated, whereby nucleation of dislocations occurred along the loading axis at an ever-increasing depth which scaled with the contact half-width $a$. For this loading profile, the surface deformation did not contribute to dislocation activity in a way 
a
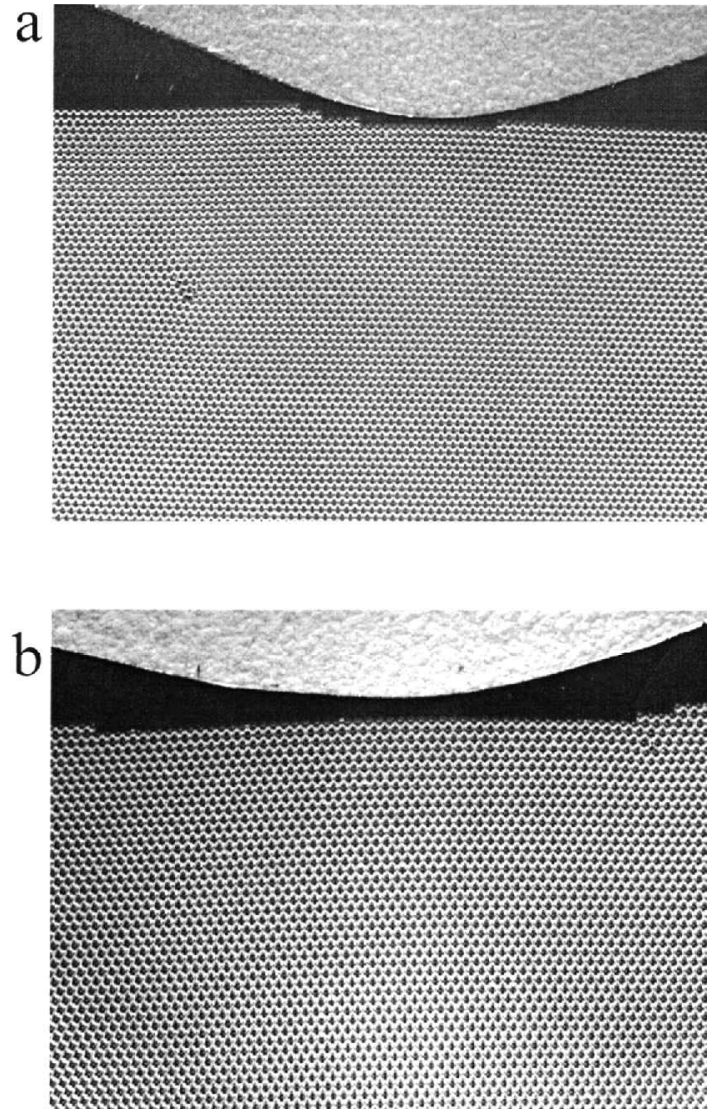

Figure 3. Surface deformation after three complete indentation cycles for indenter radii of (a) $14 \mathrm{~nm}$ and (b) $28 \mathrm{~nm}$. The dislocation nucleation event during each cycle resulted in the formation of a single slip step for each dislocation dipole. Note that, in $(a)$, two dipole nucleation events occurred in the second cycle, resulting in a total of four slip steps for 3 cycles.

that suppressed nucleation within the crystal. No dislocation nucleation was observed to initiate at the surface for the range of indenter radii and number of subsurface nucleation events observed. Upon complete unloading after these multiple nucleation events, the surface deformation resembled that of the cyclic loading experiments; slip steps of a fixed height appeared at increasing distance from the loading axis such that the surface depression conformed to the shape of the indenter tip.

The role of surface defects in effectively softening the crystal during cyclic loading is, of course, a strong function of the initial microstructure. Cyclic loading of 'polycrystalline' rafts was conducted for an indenter radius of $28 \mathrm{~nm}$. As shown in figure 4, the surface of such rafts was initially rough on the atomic scale. However, as the grain boundaries acted as dislocation sinks, surface-nucleated defects moved quickly to the boundaries, thereby facilitating grain-boundary migration. For this reason, the indented 'polycrystals' actually showed a reduction in surface roughness after the first loading cycle. Subsequent cycles (not shown) repeated this process, whereby near-surface defect nucleation and migration led to grain boundary motion. Here, we note that grain boundary motion in a real three-dimensional crystal would 
a
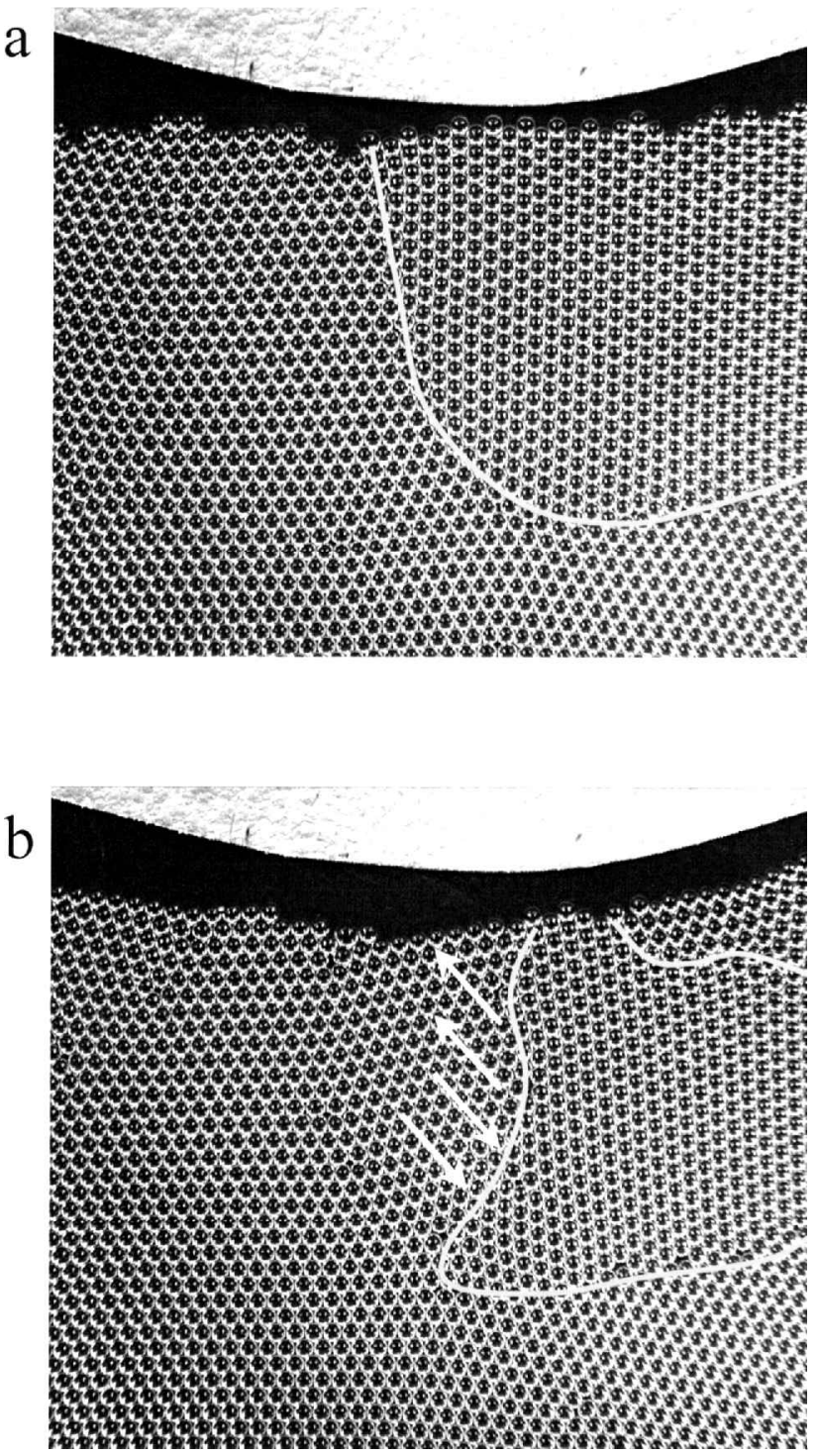

Figure 4. Indentation loading of a grain boundary, for an indenter radius of $28 \mathrm{~nm}$. (a) Atomic roughness at the free surface. $(b)$ Dislocation motion from the surface and the grain boundary leads to grain-boundary migration and a reduction in surface roughness.

be much more constrained owing to an increase in dislocation interactions and a relative reduction in free surface area.

\section{§4. Discussion}

The position of defect nucleation beneath the nominally sharp indenters investigated in these experiments was strongly affected by the loading profile. During cyclic loading, complete unloading of the crystal resulted in the formation of welldefined slip steps at the surface. These slip steps acted as points of stress concentration, undergoing immediate plastic deformation and emitting dislocations into the 
crystal upon subsequent reloading of the surface. In contrast, defects that moved to the surface during monotonic loading did not act as dislocation sources. It is thus evident that there is a contact fatigue response under indentation that is distinctly different from the more widely studied monotonic response. It appears that the fatigue response includes the suppression of homogeneous defect nucleation within the crystal, effectively causing the crystal to yield at lower loads. Because of the strong interaction of these closely spaced surface defects, however, this suppression of homogeneous nucleation may lead to increased dislocation interactions near the indented surface, resulting in an effective cyclic strain hardening of the crystal.

These observations may also elucidate the general mechanism of surface roughening during contact fatigue. The slip steps that formed and persisted under repeated loading at the surface of this two-dimensional system are reminiscent of the threedimensional extrusions that are experimentally observed to form on initially smooth free surfaces during fatigue of ductile crystals, although the geometric, crystallographic and deformation characteristics of the present slip steps and the surface roughness features of cyclically deformed metals are different. In fact, twodimensional molecular dynamics (MD) simulations of indentation have been conducted. These computational simulations quantify the motion of particles (such as bubbles or atoms) according to a given interparticle potential. The resulting predictions of defect nucleation conditions have been compared with the present bubble raft experiments (Van Vliet et al. 2002).

On the basis of such comparisons with experiments, full three-dimensional MD simulations of indentations have also been conducted. Such three-dimensional computational simulations clearly show the correspondence between contact-induced dislocation nucleation and surface slip steps in fcc crystals such as $\mathrm{Al}$ and $\mathrm{Cu}(\mathrm{Li}$ et al. 2002), consistent with the present cyclic indentation experiments. For example, MD simulations of spherical indentation in the $\langle 111\rangle$ direction in single crystal show dislocation activity on three equivalent $\{111\}\langle 110\rangle$ slip systems oriented $35.3^{\circ}$ from the loading axis. Because of the relative elastic isotropy of aluminium, dislocations nucleate on each of these slip systems simultaneously and glide to the free surface. This intersection with the free surface produces slip steps of the $\langle 110\rangle$ Burgers vector orientation on the indented $\{111\}$ plane ( $\mathrm{Li}$ et al. 2002). Upon reloading of this configuration, these slip steps would act as stress concentrations and emit dislocations from the surface into the crystal. Of course, interactions between internally nucleated dislocations would be more pronounced in three dimensions because the true two-dimensional nature of these line defects would promote jog and kink formation. In fact, it is possible that these internal interactions may dominate over the surface roughness-induced softening of the crystal during cyclic indentation. Thus, the Bragg-Nye system provides an opportunity not only for visualization and systematic investigation of this surface roughening phenomenon, but also for separation of the effects of surface roughness and dislocation interaction on the cyclic indentation response. Further extension of this work would inevitably involve observations of surface roughening characteristics over large numbers of fatigue cycles.

\section{$\S 5$. CONCLusions}

An extension of the Bragg-Nye bubble raft model has been proposed to examine the differences between cyclic and monotonic loading. In the two-dimensional model, the presence of well-formed slip steps at the surface of a cyclically loaded 
crystal significantly affects the extent of homogeneous dislocation nucleation within the crystal and confines all dislocation nucleation activity to the surface in only a few cycles. These observations also provide insights for the design of surface contact fatigue experiments, as well as MD simulations. In addition, this model may serve as a useful tool in examining the evolution of surface roughness during contact fatigue.

\section{ACKNOWLEDGEMENTS}

The authors gratefully acknowledge support of this work by the Defense University Research Initiative on NanoTechnology which is funded at the Massachusetts Institute of Technology (MIT) by the Office of Naval Research under grant N00014-01-1-0808. K.J.V.V. acknowledges support from the US Department of Defense National Defense Science and Engineering Graduate Fellowship at MIT. We also especially thank Ms Sedina Tsikata for her cheerful assistance with these experiments.

\section{REFERENCES}

Bragg, L., and Lomer, W. M., 1948, Proc. R. Soc. A, 196, 171.

Bragg, L., and Nye, J. F., 1947, Proc. R. Soc. A, 190, 474.

Corcoran, S. G., Colton, R. J., Lilleodden, E. T., and Gerberich, W. W., 1997, Phys. Rev. B, 55, 16057.

Gouldstone, A., Koh, H.-J., Zeng, K.-Y., Giannakopoulos, A. E., and Suresh, S., 2000, Acta Mater., 48, 2277.

Gouldstone, A., Van Vliet, K. J., and Suresh, S., 2001, Nature, 411, 656.

Johnson, K. L., 1985, Contact Mechanics (Cambridge University Press).

Li, J., VAn Vliet, K. J., Zhu, T., Suresh, S., and YiP, S., 2002 (submitted).

Suresh, S., 1998, Fatigue of Materials, second edition (Cambridge University Press), pp. 39-164.

Suresh, S., Nieh, T. G., and Choi, B. W., 1999, Scripta mater., 41, 951.

VAn Vliet, K. J., Li, J., Yip, S., and Suresh, S., 2002 (submitted). 